\title{
Effectiveness of Trastuzumab in First-Line HER2+ Metastatic Breast Cancer After Failure in Adjuvant Setting: A Controlled Cohort Study
}

\author{
Eva Negri, ${ }^{a}$ Alberto Zambelli, ${ }^{b}$ Matteo Franchi, ${ }^{a}$ Marta Rossi, ${ }^{a}$ Martina Bonifazi, ${ }^{a}$ Giovanni Corrao, ${ }^{c}$ Lorenzo Moja, ${ }^{\text {d,e }}$ \\ CARLo Zocchetti, ${ }^{f}$ CARLo LA Vecchia ${ }^{g}$ \\ aDepartment of Epidemiology, Istituto di Ricovero e Cura a Carattere Scientifico (IRCCS) Istituto di Ricerche Farmacologiche "Mario Negri," \\ Milan, Italy; ${ }^{b}$ Unità Strutturale Complessa (USC) Oncologia, Ospedale Papa Giovanni XXIII, Bergamo, Italy; ${ }^{\circ}$ Department of Statistics and \\ Quantitative Methods, Unit of Biostatistics and Epidemiology, University of Milano-Bicocca, Milan, Italy; ${ }^{\mathrm{d} D e p a r t m e n t}$ of Biomedical

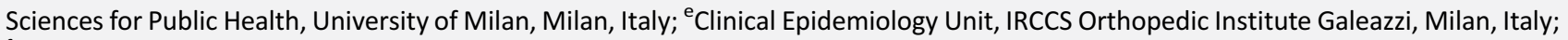 \\ ${ }^{f}$ Operative Unit of Territorial Health Service, Health Directorate, Regione Lombardia, Milan, Italy; ${ }^{\mathrm{g} D e p a r t m e n t}$ of Clinical Sciences and \\ Community Health, University of Milan, Milan, Italy \\ Disclosures of potential conflicts of interest may be found at the end of this article.
}

Key Words. Metastatic breast cancer • Overall survival • Record linkage - Trastuzumab

\section{ABSTRACT}

Background. The evidence supporting the use of trastuzumab (T) in a metastatic setting comes from studies that included (almost) only patients who never received prior T. We investigated the effectiveness of $\mathrm{T}$ as first-line therapy for metastatic breast cancer $(\mathrm{mBC})$ in women previously treated with $\mathrm{T}$ in the adjuvant setting.

Materials and Methods. By using record linkage of five administrative health care databases of Lombardy, Italy, we identified 2,046 women treated with T for early breast cancer (eBC) in 2006-2009, 96 of whom developed a metastasis and were retreated with $T$ in first-line treatment for $\mathrm{mBC}$ (treatment group). We compared the overall survival (OS) of these women with that of 197 women treated with T in firstline treatment for $\mathrm{mBC}$, who were treated with therapies other than $\mathrm{T}$ for early disease (control group). We computed
Kaplan-Meier 2-year OS and used a proportional hazard model to estimate the multivariate hazard ratio $(\mathrm{HR})$ of death in the intervention group compared with the control group, adjusted by age, use of endocrine therapy, and site of metastasis.

Results. Two-year OS was $60.0 \%$ in the treatment group and $59.5 \%$ in the control group. The adjusted HR of death in the treatment group compared with the control group was 0.79 (95\% confidence interval, 0.50-1.26).

Conclusion. Our data provide convincing evidence that the outcome of women receiving first-line $T$ treatment for $\mathrm{mBC}$ after $T$ failure in the adjuvant setting is comparable to that of women not receiving $\mathrm{T}$ for $\mathrm{eBC}$. These data are of specific interest, given the unavailability of data from randomized clinical trials. The Oncologist 2014;19:1209-1215

Implications for Practice: Trastuzumab $(T)$ is the standard of care for HER2 + breast cancer in metastatic and early stages, but scant evidence is available on its effectiveness as first-line metastatic treatment in women who failed adjuvant T. Our findings that 2-year overall survival of women treated with $\mathrm{T}$ for early breast cancer and subsequently for metastatic disease is comparable to that of women treated with Tonly for metastatic disease suggest that Tremains effective even after failure in early disease.

\section{INTRODUCTION}

HER2 overexpression occurs in $15 \%-20 \%$ of patients with breast cancer and is associated with aggressive disease and decreased survival [1]. A number of therapeutic approaches have been developed against HER2, including monoclonal antibodies and small molecule tyrosine kinase inhibitors. Trastuzumab $(T)$, the first anti-HER2 agent, is a humanized monoclonal antibody that binds to the extracellular, juxtamembrane portion of the HER2 receptor and suppresses HER2 signaling activity, resulting in inhibition of downstream signaling pathways, cell cycle arrest, and a reduction in angiogenesis [2]. In patients with HER2-amplified metastatic breast cancer $(m B C)$, Thas antitumor activity $[3,4]$, improving survival in the first-line setting when combined with chemotherapy $[5,6]$. The administration of $T$ in the initial postoperative (adjuvant) setting, in combination and/or sequentially after chemotherapy, results in an improvement in disease-free survival, with a $50 \%$ reduction in the risk of relapse, and in overall survival (OS) of patients with HER2-positive early breast cancer $(\mathrm{eBC})[7,8]$.

Despite the advances that have been brought by $\mathrm{T}$, a not negligible group of patients with HER2-positive eBC who received adjuvant $T$ therapy will eventually experience disease 
recurrence and will be proposed for treatment in the metastatic setting. The robust evidence supporting the use of T in adjuvant and metastatic settings comes from studies that primarily included only patients who never received prior T. Limited evidence supports the use of $\mathrm{T}$ beyond progression in metastatic disease [9-11], and no evidence supports its use in patients with a first relapse who failed adjuvant T. Although it is now possible to consider different options (lapatinib, pertuzumab, trastuzumab emtansine) in patients with $\mathrm{mBC}$ experiencing progression of disease while taking $\mathrm{T}$, limited approaches have been tested for patients who relapse after adjuvant $T$ [12], and the optimal strategy of therapy for this growing group of patients remains elusive. However, because $T$ also has been licensed for $\mathrm{eBC}$, even in the absence of definite evidence, the daily clinical practice has addressed the problem, and in many cases patients relapsing after adjuvant Tobtained retreatment, mostly in combination with chemotherapy and endocrine therapy, with variable and unpredictable outcomes.

Therefore, it is important to ascertain the role of $\mathrm{T}$ after failure in an adjuvant setting. In a large cohort of patients who received $\mathrm{T}$ for first-line treatment of metastatic disease, we compare the OS of women who received or did not receive $T$ in an adjuvant setting.

\section{Materials And Methods}

The Italian National Health Service (NHS) provides universal coverage with standard care to all Italian citizens. Reimbursement to hospitals and other health care providers is performed through administrative health care databases at the regional level. T was first approved in Italy for HER2 + $\mathrm{mBC}$ and in July 2006 for eBC [13]. T is dispensed by hospital pharmacies only, and since January 2006 in Lombardy, it is mandatory to register each dispensation in a special file (File F). In previous work, we built a cohort of more than 2,000 women who had used T for eBC by record linkage of several regional administrative health care databases, and we analyzed cardiotoxicity [14] and estimated OS and progression-free survival $[15,16]$ in clinical practice.

\section{Data Sources}

Five regional health administrative databases were used: (a) the File F registry 2006-2009, in which any administration of T reimbursed by the NHS has been mandatorily recorded since 2006; (b) the regional hospital discharge forms (Scheda di Dimissione Ospedaliera [SDO]) database (1997-2010); (c) the Outpatients' Services database (2002-2010); (d) the Registry Office database of Lombardy, updated to April 2011; and (e) the drug prescription database.

\section{Patient Identification by the Same Unique Anonymous Code in All Databases}

A more detailed description of the databases used for the record linkage and of the operative procedures used to identify the various groups of women are given in supplemental Appendix 1 and previous publications $[14,16]$.

\section{Selection of Intervention and Control Groups}

We carried out a computerized record linkage through the unique anonymous patient identification code. We selected all women who resided in Lombardy, who were first treated with T between August 2006 and December 2009, and who had a SDO reporting a breast cancer diagnosis before the first $T$ administration. By comparing the dates of the first SDO reporting a breast cancer diagnosis, the first SDO reporting a metastasis, and the first $T$ administration, we divided them into (a) women first treated with $\mathrm{T}$ for $\mathrm{eBC}$ and (b) women treated with $\mathrm{T}$ for $\mathrm{mBC}$ only. We excluded women for whom we could not determine whether the first $T$ treatment was for $\mathrm{eBC}$ or $\mathrm{mBC}$ (supplemental Appendix 1 shows the operative definitions).

Among women treated with $\mathrm{T}$ for $\mathrm{eBC}$, we identified those who developed a metastasis and restarted or continued $T$ treatment after the metastasis. We further excluded women who did not receive $\mathrm{T}$ for $\mathrm{mBC}$ as first-line treatment. Therefore, the "treatment group" consisted of women who were treated with $\mathrm{T}$ for $\mathrm{EBC}$ and were treated again with $\mathrm{T}$ as first-line therapy (supplemental Appendix 1 shows the operative definitions) for $\mathrm{mBC}$ ( $\mathrm{mBC} / \mathrm{adj}+$ ) .

For the control group, we selected patients treated with $\mathrm{T}$ for $\mathrm{mBC}$ only. We excluded patients with "up-front metastatic disease" from the control group, that is, women who were not previously treated for $\mathrm{eBC}$ with a therapy other than $\mathrm{T}$. We also excluded women treated with $\mathrm{T}$ for $\mathrm{mBC}$, but not as first-line therapy. Therefore, the "control group" consisted of women who were treated for $\mathrm{eBC}$, but not with $\mathrm{T}$, and were treated with $\mathrm{T}$ as first-line therapy for $\mathrm{mBC}$ ( $\mathrm{mBC} /$ adjT-).

\section{Statistical Analysis}

The OS was estimated using the Kaplan-Meier method [17] and was defined as the time from the first trastuzumab prescription for $\mathrm{mBC}$ to death from any cause (from the SDO database and/ or the Registry Office database). Patients were followed up until death or April 30, 2011, whichever came first. Differences in OS curves were tested by the log-rank test [17].

We also estimated the hazard ratio (HR) of death of women previously treated with $\mathrm{T}$ for $\mathrm{eBC}(\mathrm{mBC} / \mathrm{adj} \mathrm{T}+)$ compared with women treated with $\mathrm{T}$ for $\mathrm{mBC}$ only ( $\mathrm{mBC} /$ adjT - ), adjusted by age ( $<50,50-59,60-69,70+$ years), use of endocrine therapy, and site of metastasis (brain, liver/lung, other), using Cox proportional hazards models [17]. The proportional hazard assumption was assessed by both plotting minus $\log (\log$ (survival function)) versus log(time) and using the analytic method based on comparison between observed residuals and a randomly generated sample process (Kolmogorov-type supremum test) [17].

We also compared the survival of women treated with $\mathrm{T}$ for $\mathrm{eBC}$ who did and did not restart or continue the treatment for the metastatic disease. In this case, OS was defined as the time from the diagnosis of metastasis to death from any cause or end of follow-up.

\section{RESULTS}

Figure 1 presents a flowchart of the selection of the treatment and control groups. Overall, 2,879 patients received the first T prescription between August 2006 and December 2009. We excluded 9 men and 143 women with inconsistent data, or for whom we could not define whether T was first used for $\mathrm{eBC}$ or $\mathrm{mBC}$. Of the 2,046 women first treated with $\mathrm{T}$ for eBC, 240 developed distant recurrence after the beginning of treatment; 113 did not use $T$ after the diagnosis of metastasis, whereas 127 continued or restarted the therapy, 96 of whom received T as first-line treatment for $\mathrm{mBC}$ ("treatment group," 


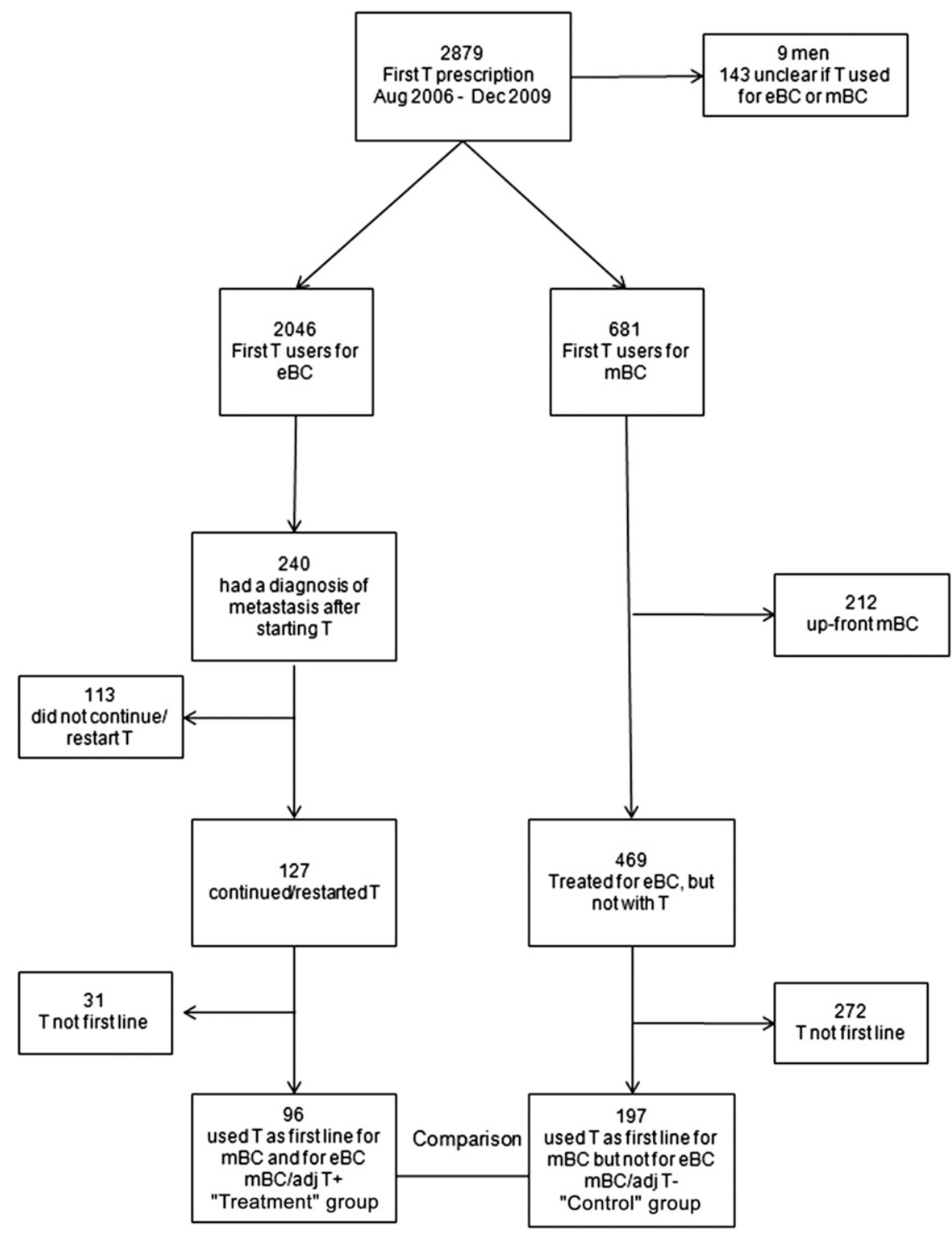

Figure 1. Flowchart describing the selection of the treatment and control groups from the cohort of women treated with T for breast cancer.

Abbreviations: AdjT, adjuvant trastuzumab; eBC, early breast cancer; mBC, metastatic breast cancer; $\mathrm{T}$, trastuzumab.

$\mathrm{mBC} / \mathrm{adjT}+)$. Compared with women who continued or restarted $\mathrm{T}(n=127)$, those who did not receive T after the diagnosis of the metastasis $(n=113)$ were older $(26.6 \%$ vs. $10.2 \%$ aged $\geq 70$ years) and more frequently had brain metastases (34.5\% vs. 9.5\%) (supplemental online Table 1). One year after metastasis, OS was $39.8 \%$ in women who did not restart $\mathrm{T}$ and $88.0 \%$ in women who did. In the 96 women included in the treatment group, 64 (67\%) of the metastases occurred during or within 6 months since stopping T treatment.

A total of 681 women first received $T$ for $\mathrm{mBC}$ only. After exclusion of 212 women who had "up-front metastatic disease" and a further 272 women who received T for $\mathrm{mBC}$ not as first-line therapy, the control group included 197 women who received $T$ as first-line treatment for $\mathrm{mBC}$ and were treated with therapies other than $\mathrm{T}$ for $\mathrm{eBC}(\mathrm{mBC} / \mathrm{adj} \mathrm{T}-$ ).

Table 1 shows the age and the site of metastasis of $96 \mathrm{~T}$ users for $\mathrm{eBC}$ and subsequent first-line treatment for $\mathrm{mBC}$ (mBC/adjT + , treatment group) and of 197 T users for first-line treatment for $\mathrm{mBC}$ only ( $\mathrm{mBC} / \mathrm{adj} \mathrm{T}-$, control group), excluding women with "up-front metastatic disease." Women in the treatment group were younger $(41.7 \%$ vs. $23.8 \%$ aged $<50$ years) and more frequently had brain metastases (9.4\% vs. 4.1\%) than women in the control group. The median follow-up was 2.5 years. Survival estimates for the treatment group were $87.5 \%$ after 1 year of follow-up and $60.0 \%$ after 2 years of follow-up; corresponding figures for the control group were $80.6 \%$ and $59.5 \%$, respectively (Fig. 2 ).

Table 2 presents the results of the proportional hazard model, including terms for age, use of endocrine therapy, metastatic site, and treatment/control group. No significant difference emerged with age. Compared with women with brain metastases, those with liver and lung metastases had a lower hazard of dying ( $\mathrm{HR}, 0.28 ; 95 \%$ confidence interval $[\mathrm{Cl}]$, 0.14-0.54), and those with only metastases at sites other than the brain, liver, or lung had an even lower risk (HR, $0.13 ; 95 \% \mathrm{Cl}$, 0.07-0.27). The HR of death in the treatment group (mBC/ $\mathrm{adj}+$ ) compared with the control group (mBC/adjT-), 
Table 1. Age, use of endocrine therapy, and site of metastasis among 96 women treated with $T$ for both early breast cancer (eBC) and $\mathrm{mBC}$ as first line of therapy ( $\mathrm{mBC} / \mathrm{adj} \mathrm{T}+$ ), and 197 women treated with other therapies for $\mathrm{EBC}$ and first-line $\mathrm{T}$ therapy for $\mathrm{mBC}$ (mBC/adjT-)

\begin{tabular}{|c|c|c|c|c|}
\hline & \multicolumn{2}{|c|}{$\mathrm{mBC} / \mathrm{adj} T+96$} & \multicolumn{2}{|c|}{ mBC/adjT- 197} \\
\hline & $n$ & $\%$ & $n$ & $\%$ \\
\hline \multicolumn{5}{|l|}{$\operatorname{Age}^{a}$ (yrs) } \\
\hline$<40$ & 11 & 11.5 & 16 & 8.1 \\
\hline $40-49$ & 29 & 30.2 & 31 & 15.7 \\
\hline $50-59$ & 25 & 26.0 & 48 & 24.4 \\
\hline $60-69$ & 20 & 20.8 & 57 & 28.9 \\
\hline$\geq 70$ & 11 & 11.5 & 45 & 22.8 \\
\hline$p$ for heterogeneity & .009 & & & \\
\hline \multicolumn{5}{|c|}{ Use of endocrine therapy } \\
\hline No & 54 & 56.3 & 88 & 44.7 \\
\hline Yes & 42 & 43.7 & 109 & 55.3 \\
\hline$p$ for heterogeneity & .063 & & & \\
\hline \multicolumn{5}{|l|}{ Metastatic site } \\
\hline Brain & 9 & 9.4 & 8 & 4.1 \\
\hline Liver/lung & 42 & 43.7 & 107 & 54.3 \\
\hline Other & 45 & 46.9 & 82 & 41.6 \\
\hline$p$ for heterogeneity & .083 & & & \\
\hline
\end{tabular}

${ }^{\mathrm{a}} \mathrm{Age}$ at first trastuzumab prescription for metastatic breast cancer. Abbreviations: AdjT, adjuvant trastuzumab; $\mathrm{mBC}$, metastatic breast cancer.

adjusted by age, history of endocrine therapy, and metastatic site, was $0.79(95 \% \mathrm{Cl}, 0.50-1.26)$, similar to the unadjusted $\mathrm{HR}$ (0.86; 95\% Cl, 0.55-1.34).

\section{DisCUSSION}

Our study reports the OS of a cohort of patients with $\mathrm{mBC}$, treated with first-line T-based combination therapy, who received or did not receive $T$ in an adjuvant setting. Of 293 patients with $\mathrm{MBC}$ who received $\mathrm{T}$ as first-line treatment, the 96 women treated with $\mathrm{T}$ for $\mathrm{eBC}$ and retreated for metastatic disease showed an OS that was similar to the 197 women who received $\mathrm{T}$ for $\mathrm{mBC}$ only, that is, not treated with $\mathrm{T}$ before the diagnosis of metastasis ( $60 \%$ in both groups after 2 years; HR of death, $0.79 ; 95 \% \mathrm{Cl}, 0.50-1.26)$.

$T$ is the well-established mainstay treatment of women affected by HER2 + breast cancer. The effectiveness of T has been widely demonstrated in both adjuvant and metastatic HER2 + breast cancer, and some observations suggested a potential role of $\mathrm{T}$ beyond disease progression $[9-11,18]$. According to this evidence, Thas been predicted to be effective in the first-line setting of $\mathrm{mBC}$ after T adjuvant failure. Although novel potential anti-HER2 options have been developed, at the present time, the retreatment of patients with metastatic cancer with T still represents a widely used option, notwithstanding that the clinical impact of such a strategy has never been formally ascertained in controlled randomized clinical trials (RCTs).

The Italian guidelines for breast cancer treatment (Associazione Italiana di Oncologia Medica [AIOM] 2012) [19] advise to restart $\mathrm{T}$ in association with chemotherapy (taxanes, vinorelbine, capecitabine) as first-line treatment of $\mathrm{mBC}$ in women treated with $\mathrm{T}$ in the adjuvant setting. The level of evidence of this recommendation is rated on the basis of expert opinion alone, that is, without any support from analytic studies, and the strength of this recommendation is rated as weak positive, adapted from the GRADE method [20]. The U.S. National Comprehensive Cancer Network (NCCN) guidelines accept taxotere, trastuzumab, and pertuzumab as the preferred first-line therapy for patients with metastatic HER2 +, including patients who received prior trastuzumab in the adjuvant setting (NCCN 2014) [21]. The National Institute for Health and Clinical Excellence 2009 guidelines [22] do not specifically address the problem, but suggest discontinuation of $\mathrm{T}$ for $\mathrm{mBC}$ in case of disease progression outside the central nervous system. Thus, there are different attitudes, reflecting the lack of solid data on the matter, in the absence of information generated from dedicated clinical trials. At the same time, it is unlikely that such trials will be launched in the near future because of the evident ethical and clinical concerns these investigations would implicate. Consequently, information in this field is mostly confined to retrospective studies and post hoc subgroup analyses of existing datasets.

This uncertainty also is reflected by the fact that of the 240 women who developed a metastasis while treated with $\mathrm{T}$ for eBC, 127 (53\%) were retreated with T and 113 (47\%) were not. This is not surprising given the lack of solid evidence about Tuse in this context, particularly at the time of the study. Older age, comorbidities, and the presence of a brain metastasis were strong predictors for not receiving $\mathrm{T}$ for $\mathrm{mBC}$ after failure in the adjuvant setting in our data. There is strong evidence that older patients are undertreated [23]. Moreover, the cardiotoxicity of $\mathrm{T}$ [14] has likely discouraged its use in women at high risk of cardiac disease, as did the presence of a brain metastasis, for which the use of $T$ has been debated $[24,25]$.

An issue to bear in mind is that because of the short timeframe of this study and the introduction of adjuvant Tonly in mid-2006 in Italy, two thirds of the women who received T for both $\mathrm{eBC}$ and $\mathrm{mBC}$ were refractory to $\mathrm{T}$, that is, developed a metastasis during $T$ treatment or within 6 months from stopping $T$ treatment. Thus, our cohort is mostly composed of women for whom the response to $T$ in the adjuvant setting has been worse, at least in terms of efficacy. Thus, strictly speaking, our results do not apply to women who develop a metastasis long after stopping $\mathrm{T}$ for $\mathrm{eBC}$, although these women should, if anything, have a better prognosis than the women in our treatment group.

The control group was not treated with the most effective therapy in the beginning. However, the use of $T$ in the adjuvant setting was authorized in Italy in mid-2006; thus, women with a HER2 + early breast cancer in the few years before that date were not treated with T. Moreover, T-naïve women are exactly those for whom the efficacy of $\mathrm{T}$ for $\mathrm{mBC}$ has been demonstrated.

Our data are consistent with those recently reported in two RCTs, partially focusing on the same theme as our study. First, the CLEOPATRA RCT, while comparing first-line pertuzumab, trastuzumab, and docetaxel with placebo, trastuzumab, and docetaxel for HER2 + $\mathrm{mBC}$, included 80 patients with $\mathrm{mBC}$ ( $10 \%$ of the entire study population) in the analysis who were treated with $T$ in the neoadjuvant/adjuvant setting [26]. In 


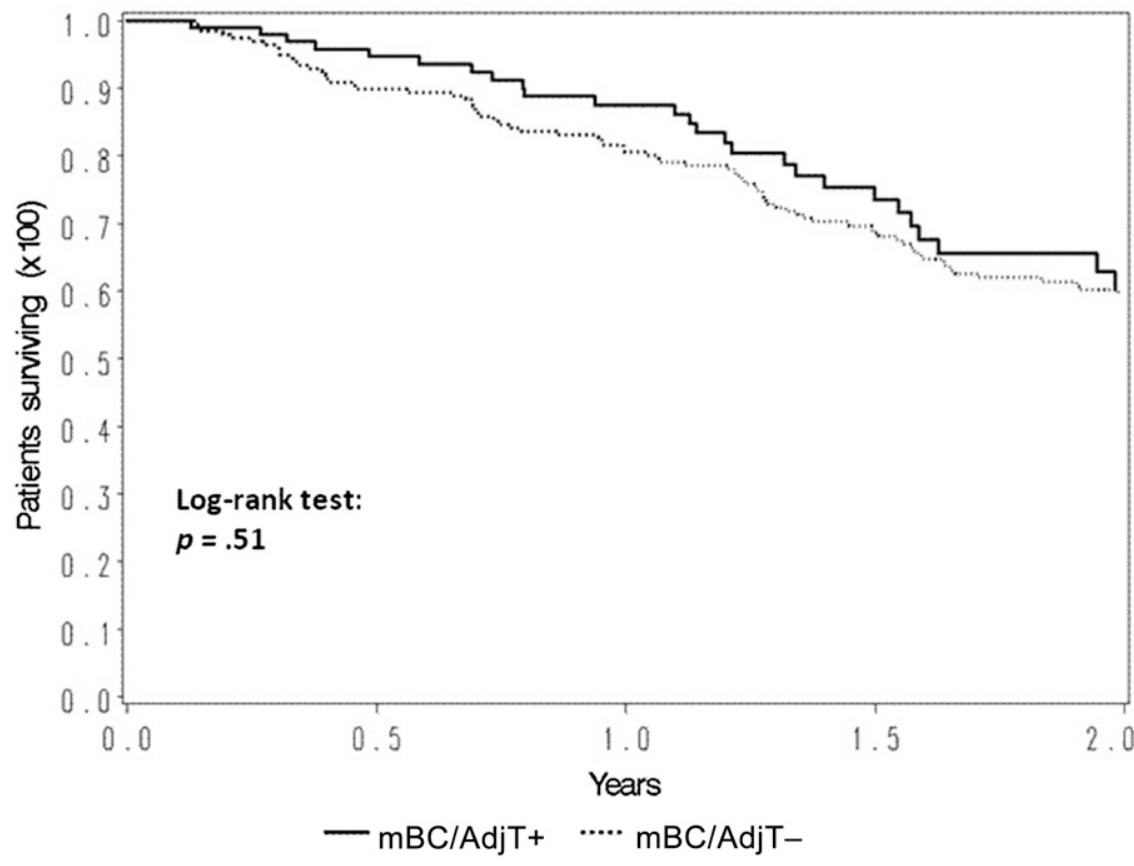

mBC/Adj T+

$\begin{array}{rrr}\text { No. at risk } & 68 & 21 \\ \text { Survival (\%) } & 87.5 & 60.0 \\ & & \\ \text { No. at risk } & 156 & 95 \\ \text { Survival (\%) } & 80.6 & 59.5\end{array}$

Figure 2. Kaplan-Meier estimates of overall survival among 96 women treated with $\mathrm{T}$ for both eBC and $\mathrm{mBC}$ as first line of therapy (mBC/ $\mathrm{adjT}+$ ) and 197 women treated with other therapies for $\mathrm{eBC}$ and first-line $\mathrm{T}$ therapy for $\mathrm{mBC}$ (mBC/adjT-).

Abbreviations: AdjT, adjuvant trastuzumab; eBC, early breast cancer; $\mathrm{mBc}$, metastatic breast cancer.

Table 2. Estimated $\mathrm{HR}$ and corresponding $95 \% \mathrm{Cls}$ from multivariate Cox model among 197 women treated with other therapies for early breast cancer (eBC) and first-line T therapy for $\mathrm{mBC}(\mathrm{mBC} / \mathrm{adj} \mathrm{T}-$ ), and 96 women treated with $\mathrm{T}$ for both eBC and $\mathrm{mBC}$ as first line of therapy $(\mathrm{mBC} / \mathrm{adj} \mathrm{T}+)$

\begin{tabular}{|c|c|c|c|}
\hline & No. & No. (\%) of events & $\mathrm{HR}^{\mathrm{a}}(95 \% \mathrm{Cl})$ \\
\hline \multicolumn{4}{|l|}{ Group } \\
\hline $\mathrm{mBC} / \mathrm{adj} \mathrm{T}-$ & 197 & $77(39.1)$ & $1^{\mathrm{b}}$ \\
\hline $\mathrm{mBC} / \mathrm{adj}++$ & 96 & $26(27.1)$ & $0.79(0.50-1.26)$ \\
\hline \multicolumn{4}{|l|}{ Age, yrs } \\
\hline$<70$ & 237 & $82(34.6)$ & $1^{\mathrm{b}}$ \\
\hline$\geq 70$ & 56 & $21(37.5)$ & $1.16(0.98-1.37)$ \\
\hline \multicolumn{4}{|c|}{ Use of endocrine therapy } \\
\hline No & 142 & $63(44.4)$ & $1^{\mathrm{b}}$ \\
\hline Yes & 151 & $70(46.4)$ & $0.87(0.59-1.29)$ \\
\hline \multicolumn{4}{|l|}{ Metastatic site } \\
\hline Brain & 17 & $12(70.6)$ & $1^{\mathrm{b}}$ \\
\hline Liver/lung & 149 & $63(42.3)$ & $0.28(0.14-0.54)$ \\
\hline Other & 127 & $28(22.1)$ & $0.13(0.07-0.27)$ \\
\hline
\end{tabular}

\footnotetext{
${ }^{a}$ Estimated by multivariate Cox model adjusted by intervention group, age, use of endocrine therapy, and metastatic site.

${ }^{\mathrm{b}}$ Reference category.

Abbreviations: AdjT, adjuvant trastuzumab; $\mathrm{Cl}$, confidence interval; $\mathrm{HR}$, hazard ratio; $\mathrm{mBC}$, metastatic breast cancer.
}

a post hoc subgroup analysis, the favorable effect on OS of adding pertuzumab to $\mathrm{T}$ and docetaxel in women previously treated with $\mathrm{T}(\mathrm{HR}, 0.68 ; 95 \% \mathrm{Cl}, 0.30-1.55)$ was similar to that for the whole intention-to-treat population $(\mathrm{HR}, 0.69 ; 95 \% \mathrm{Cl}$, 0.58-0.81). However, the authors did not compare the OS of women previously treated with $\mathrm{T}$ with those who were not. 
Second, a nonrandomized, multicenter, open-label phase II study in mBC (Retreatment after HErceptin Adjuvant [RHEA] trial) reported an encouraging activity of $T$ in previously treated eBC [27]. Indeed, the RHEA study investigated the efficacy and safety of $T$ plus a taxane in patients who experienced a relapse after adjuvant $\mathrm{T}$. For the 41 included patients, partial response was observed in 25 (61\%), and median progression-free survival was 8.0 months [27]. To the best of our knowledge, this retrospective analysis represents the most extensive study exploring the effectiveness of $\mathrm{T}$ after adjuvant failure.

Given the lack of data from RCTs on this issue, our study offers a valuable contribution, despite its limitations. Our study is based on record linkage of administrative databases, and therefore it is conceivable that some misclassification in defining the various groups has occurred. The fact that our cohort of women treated with $\mathrm{T}$ for $\mathrm{EBC}$ had an OS comparable to that of the HERceptin Adjuvant trial $[16,28]$ is reassuring against substantial misclassification of $T$ treatment for $\mathrm{mBC}$ as $\mathrm{eBC}$. Another limitation is the lack of detailed clinical information, which also prevented the evaluation of progression-free survival in our cohort of patients.

Moreover, given the "real-world" nature of our data [15, 29], the modes of patients' metastatic workup at diagnosis and follow-up were heterogeneous. The Italian guidelines, from the National Society of Medical Oncology (AIOM), provide regular recommendations for tumor assessment at the time of the first breast cancer diagnosis. Because the population is derived exclusively from the Lombardy region, we do have reasons to expect a consistent approach in this population regarding the diagnostic metastatic workup. The retrospective nature of this observation and the sources of our database prevented us from obtaining a centralized assessment of HER2 status. However, our analysis moved from drug $(T)$ rather than cancer subtype (HER2), and the criteria for patients' enrollment were based on $\mathrm{T}$ administration. We have discussed the problem of the quality of HER2 status ascertainment in a previous article [14]. However, there is no reason why the quality of ascertainment of HER2 status should differ between our two groups. Thus, these potential limitations do not attenuate the message of T effectiveness emerging from the OS analysis. If, as the evidence from RCTs shows, $T$ is effective in T-naïve women treated for $\mathrm{mBC}$, then our results indicate that it is also effective after $\mathrm{T}$ failure in the adjuvant setting, and to approximately the same extent.

\section{CONCLUSION}

Given the lack of data from RCTs, our study provides the strongest evidence that the outcome of women receiving firstline $T$ treatment for $\mathrm{mBC}$ after $T$ failure in an adjuvant setting is comparable to that of women not exposed to T during eBC therapy.

\section{ACKNOWLEDGMENTS}

This work was conducted with contributions from the Regione Lombardia and Fondazione Umberto Veronesi, and it was partly supported by the Italian Association for Cancer Research and the Italian Foundation for Cancer Research. We thank I. Garimoldi for editorial assistance.

\section{Author Contributions}

Conception/Design: Eva Negri, Alberto Zambelli, Martina Bonifazi, Carlo La Vecchia

Provision of study material or patients: Giovanni Corrao, Carlo Zocchetti Collection and/or assembly of data: Matteo Franchi, Marta Rossi

Data analysis and interpretation: Eva Negri, Alberto Zambelli, Matteo Franchi, Marta Rossi, Martina Bonifazi, Giovanni Corrao, Lorenzo Moja, Carlo Zocchetti, Carlo La Vecchia

Manuscript writing: Eva Negri, Alberto Zambelli, Matteo Franchi, Marta Rossi, Martina Bonifazi, Carlo La Vecchia

Final approval of manuscript: Eva Negri, Alberto Zambelli, Matteo Franchi, Marta Rossi, Martina Bonifazi, Giovanni Corrao, Lorenzo Moja, Carlo Zocchetti, Carlo La Vecchia

DISCLOSURES

The authors indicated no financial relationships.

\section{REFERENCES}

1. Slamon DJ, Clark GM, Wong SG et al. Human breast cancer: Correlation of relapse and survival with amplification of the HER-2/neu oncogene. Science 1987;235:177-182.

2. Baselga J, Albanell J. Mechanism of action of anti-HER2 monoclonal antibodies. Ann Oncol 2001; 12(suppl 1):S35-S41.

3. Baselga J,Tripathy D, Mendelsohn J et al. Phase I study of weekly intravenous recombinant humanized anti-p185HER2 monoclonal antibody in patients with HER2/neu-overexpressing metastatic breast cancer. J Clin Oncol 1996;14:737-744.

4. Cobleigh MA, Vogel CL, Tripathy $D$ et al Multinational study of the efficacy and safety of humanized anti-HER2 monoclonal antibody in women who have HER2-overexpressing metastatic breast cancer that has progressed after chemotherapy for metastatic disease. J Clin Oncol 1999;17: 2639-2648.

5. Slamon DJ, Leyland-Jones B, Shak S et al. Use of chemotherapy plus a monoclonal antibody against HER2 for metastatic breast cancer that overexpresses HER2. N Engl J Med 2001;344:783-792.

6. Marty M, Cognetti F, Maraninchi D et al. Randomized phase II trial of the efficacy and safety of trastuzumab combined with docetaxel in patients with human epidermal growth factor receptor 2 positive metastatic breast cancer administered as first-line treatment: The M77001 study group. J Clin Oncol 2005;23:4265-4274.

7. Piccart-Gebhart MJ, Procter M, Leyland-Jones B et al. Trastuzumab after adjuvant chemotherapy in HER2-positive breast cancer. N Engl J Med 2005;353 1659-1672.

8. Romond EH, Perez EA, Bryant J et al. Trastuzumab plus adjuvant chemotherapy for operable HER2-positive breast cancer. N Engl J Med 2005; 353:1673-1684.

9. von Minckwitz G, du Bois A, Schmidt $M$ et al. Trastuzumab beyond progression in human epider mal growth factor receptor 2-positive advanced breast cancer: A German breast group 26/breas international group 03-05 study. J Clin Oncol 2009 27:1999-2006.

10. Blackwell KL, Burstein HJ, Storniolo AM et al. Overall survival benefit with lapatinib in combination with trastuzumab for patients with human epidermal growth factor receptor 2-positive metastatic breast cancer: Final results from the EGF104900 Study. J Clin Oncol 2012;30:2585-2592.
11. Esposito A, Munzone E, Bagnardi V et al. Are there benefits in routine clinical practice of continuing trastuzumab after progression for metastatic breast cancer patients? Anticancer Drugs 2012;23:1089-1098.

12. Baselga J, Cortés J, Kim SB et al. Pertuzumab plus trastuzumab plus docetaxel for metastatic breast cancer. N Engl J Med 2012;366:109-119.

13. Agenzia Italiana del Farmaco. Estensione delle indicazioni terapeutiche della specialita' medicinale «Herceptin» (trastuzumab), autorizzata con decisione della Commissione europea in data 22 maggio 2006 (Determinazione/C n. 100/ 2006). Available at http://www.gazzettaufficiale. it/atto/serie_generale/aricaDettaglioAtto/originario; jsessionid=tTd8CleHEbSwkmxxQ07Llw .ntc-as4 guri2a?atto.dataPubblicazioneGazzetta=2006-08 01\&atto.codiceRedazionale $=06 \mathrm{A07077}$ \&elenco30 giorni=false. Accessed October 17, 2014.

14. Bonifazi M, Franchi M, Rossi Metal.Trastuzumabrelated cardiotoxicity in early breast cancer: A cohort study. The Oncologist 2013;18:795-801.

15. Negri E, Rossi M, Bonifazi M et al. Clinical use, safety and effectiveness of novel high cost antican cer therapies after marketing approval: A record 
linkage study. Epidemiol Biostat Public Health 2013; 10:e9016.

16. Bonifazi M, Franchi M, Rossi M et al. Long term survival of HER2-positive early breast cancer treated with trastuzumab-based adjuvant regimen: A large cohort study from clinical practice. Breast 2014;23: 573-578.

17. Kalbfleish JD, Prentice RL. The Statistical Analysis of Failure Time Data. 2nd ed.Hoboken, New Jersey: John Wiley \& Sons, Inc., 2002.

18. Petrelli F, Barni S. A pooled analysis of 2618 patients treated with trastuzumab beyond progression for advanced breast cancer. Clin Breast Cancer 2013;13:81-87.

19. Associazione Italiana di Oncologia Medica. Linee Guida Neoplasie della Mammella. Available at http://www.aiom.it/C_Common/Download.asp? file=/\$Site\$/Attivita_Scientifica/Linee_Guida/2012/ LGAIOM2012_01_Mammella2.pdf. Accessed October 17, 2014.

20. Guyatt GH, Oxman AD, Vist GE et al. GRADE: An emerging consensus on rating quality of evidence and strength of recommendations. BMJ 2008;336:924-926.
21. National Comprehensive Cancer Network (NCCN) Clinical Practice Guidelines in Oncology. Breast cancer. Available at http://www.nccn.org/ professionals/physician_gls/f_guidelines.asp\#breast. Accessed October 17, 2014

22. National Institute for Health and Clinical Excellence (NICE) Clinical Guidelines. Advanced breast cancer update: Diagnosis and treatment. Available at http://www.nice.org.uk/guidance/ cg81/resources/guidance-advanced-breast-cancerupdate-pdf. Accessed October 17, 2014.

23. Mazzucco W, Rossi M, Cusimano R et al. Use of trastuzumab for breast cancer: The role of age. Curr Pharm Des 2014;20:5957-5962.

24. Pieńkowski T, Zielinski CC. Trastuzumab treatment in patients with breast cancer and metastatic CNS disease. Ann Oncol 2010;21: 917-924.

25. Pestalozzi BC, Zahrieh $\mathrm{D}$, Price $\mathrm{KN}$ et al. Identifying breast cancer patients at risk for Central Nervous System (CNS) metastases in trials of the International Breast Cancer Study Group (IBCSG). Ann Oncol 2006;17:935-944.
26. Swain SM, Kim SB, Cortés J et al. Pertuzumab, trastuzumab, and docetaxel for HER2-positive metastatic breast cancer (CLEOPATRA study): Overall survival results from a randomised, double-blind, placebo-controlled, phase 3 study. Lancet Oncol 2013;14:461-471

27. Láng I, Bell R, Feng FY et al. Trastuzumab retreatment after relapse on adjuvant trastuzumab therapy for human epidermal growth factor receptor 2-positive breast cancer: Final results of the Retreatment after HErceptin Adjuvant trial. Clin Oncol (R Coll Radiol) 2014; 26:81-89.

28. Gianni L, Dafni U, Gelber RD et al. Treatment with trastuzumab for 1 year after adjuvant chemotherapy in patients with HER2-positive early breast cancer: A 4-year follow-up of a randomised controlled trial. Lancet Oncol 2011;12 236-244.

29. Corrao G. Building reliable evidence from realworld data: Methods, cautiousness and recommendations. Epidemiol Biostat Public Health 2013;10: e8981.

\section{For Further Reading:}

Francisco J. Esteva, Sandra X. Franco, Maura K. Hagan et al. An Open-Label Safety Study of Lapatinib Plus Trastuzumab Plus Paclitaxel in First-Line HER2-Positive Metastatic Breast Cancer. The Oncologist 2013;18:661-666.

\section{Implications for Practice:}

Dual targeting of the HER2 receptor using trastuzumab and lapatinib has been shown to be effective in HER2-positive metastatic breast cancer. In this study, we evaluated the safety of paclitaxel in combination with trastuzumab and lapatinib. The main side effect was diarrhea, which occurred in the majority of patients at the standard dosing of all three drugs. A pharmacokinetic interaction was found between paclitaxel and lapatinib, resulting in increased exposure of both drugs. We evaluated three dose levels of lapatinib and paclitaxel (all patients received standard trastuzumab dosing). A dose of lapatinib $750 \mathrm{mg} /$ day had the lowest incidence of diarrhea in combination with paclitaxel $80 \mathrm{mg} / \mathrm{m}^{2}$ per week and trastuzumab $2 \mathrm{mg} / \mathrm{kg}$ per week. These doses should be used if the triplet is considered for further development in patients with HER2-positive metastatic breast cancer. 